\title{
THE ATHENA-OAWL DOPPLER WIND LIDAR MISSION
}

\author{
Sara C. Tucker $^{1 *}$, Carl Weimer ${ }^{1}$, R. Michael Hardesty ${ }^{2}$ \\ ${ }^{1}$ Ball Aerospace and Technologies Corp., Boulder, Colorado, USA, *Email: stucker@ball.com, \\ ${ }^{2}$ University of Colorado, Cooperative Institute for Research in Environmental Science, \\ Boulder, Colorado, USA
}

\begin{abstract}
With the objective of providing tropospheric wind profile data over the mid-latitude oceans and tropics for data-starved weather forecast models, the Earth Venture Instrument (EV-I) Mission concept "Atmospheric Transport, Hurricanes, and Extratropical Numerical weAther prediction with the Optical Autocovariance Wind Lidar" (ATHENA-OAWL) was proposed in November 2013. The mission concept is described here along with a brief history of the OAWL system development and current development of an ATHENA-OAWL airborne demonstrator under NASA's Venture Technology development.
\end{abstract}

\section{INTRODUCTION}

The need for and feasibility of putting a Doppler Wind Lidar (DWL) into space for global wind monitoring have been covered in multiple works, spanning four decades, from Huffaker et al. 1978[1] to Baker et al 2014[2] and Atlas et al. 2015 [3]. To date, a DWL has not yet flown in space, although ESA's Aeolus mission promises to launch in late 2016. [4][5] In the US, however, technological readiness, uncertainty about what approach would provide the most impact on weather forecasting, and projected cost have attenuated space-based Doppler wind lidar system developments.

Starting in 2003, Ball Aerospace (Ball) has been developing a direct-detection method for remote measurement of winds based on the Optical Autocovariance (OA) approach first outlined in 1995 by Schwiesow and Mayor. [6] The Ball OAWL development efforts overlapped with, and still benefit from, the design and build of the CALIOP lidar at Ball for the CALIPSO mission, still in operation today. In 2007, Ball demonstrated a proof-of-concept, 3-phase OA receiver measuring aerosol-backscattered Doppler shifts verified by a sonic anemometer [7]. From 2009-2012, Ball, with additional support from NASA's Earth Science Technology Office
(ESTO) built a full breadboard version of the four-phase Optical Autocovariance Wind Lidar (OAWL) and demonstrated and validated the system in ground and preliminary airborne, autonomous, flight tests.[8] Subsequent funding from ESTO and internal Ball investments led to additional development of the system, mission concepts, and space-based feasibility studies including: 1) an Instrument Design Lab (IDL) run at NASA Goddard to evaluate the flight readiness and provide parametric cost estimates of a preliminary concept and 2) OAWL-based Observing System Simulation Experiments (OSSEs, teaming with NOAA, NASA, JCSDA and Simpson Weather Associates).

\section{METHODOLOGY}

\subsection{Optical Autocovariance}

The Optical Autocovariance approach to measuring Doppler shifts works similar to a Michelson interferometer (which measures the autocovariance of a signal with itself). Rather than moving an arm of a Michelson interferometer to map out multiple fringes, however, we modify a Mach Zehnder interferometer using polarization to measure four points on a single fringe simultaneously. This quadrature Mach Zehnder direct detection approach [9][10][11] is enhanced with a reflective cats-eye design [(US Patents US7929215B1, US8077294B1] to provide field widening for use with large telescopes without costly wavefront quality requirements. Aside from minor coating losses, the reflective system is $100 \%$ transmissive, ensuring there are no return signal losses from filtering (such as observed with double-edge Fabry-Perot systems).

Through digitization and processing, the phases of Doppler shifted backscatter laser returns are estimated and compared to the estimated phase of the outgoing illumination. The corresponding phase shifts (frequency shift) linearly correspond to the line of sight wind measurements. The relatively-long $0.9 \mathrm{~m}$ optical path difference 
(OPD) between the two arms of the interferometer in the original OAWL system, and the proposed ATHENA-OAWL mission provides increased sensitivity to Doppler shifts from narrow-band aerosol backscatter signals, enabling groundbased line-of-sight wind speed precision values of $<1 \mathrm{~m} / \mathrm{s}$, similar to coherent detection system performance, but with the added benefit of being able to use photon-counting detection. A similar system operating at a shorter OPD [10] would be able to resolve Doppler shift measurements in the relatively broad-band molecular backscatter with the typical $2-3 \mathrm{~m} / \mathrm{s}$ precision.

With the correct reflective coatings, the OAWL system may be built to operate at any laser wavelength. As shown in Figure 1, the existing OAWL system has demonstrated simultaneous wind measurement operation at both the $355 \mathrm{~nm}$ and $532 \mathrm{~nm}$ laser wavelengths.
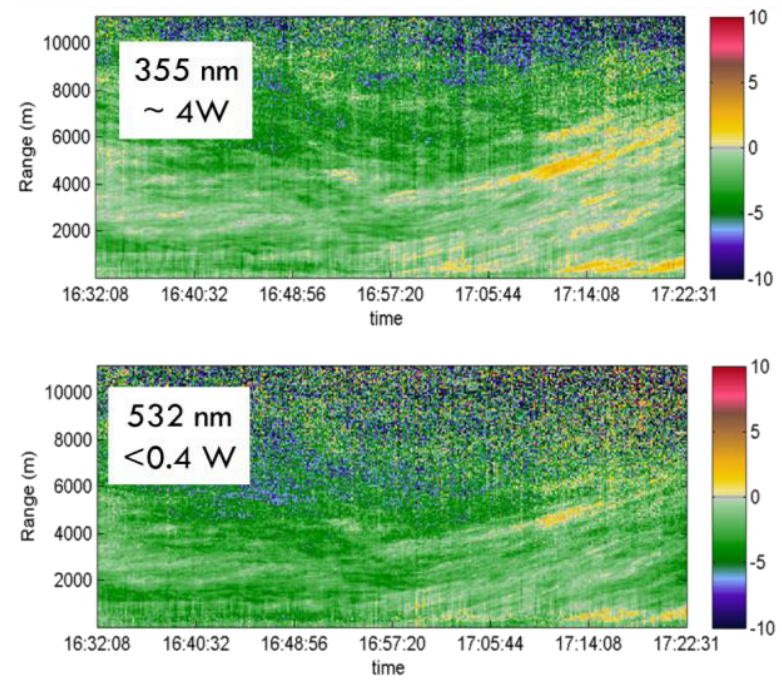

Figure 1. An example of the OAWL LOS wind speed measurements acquired simultaneously at both the 355 and $532 \mathrm{~nm}$ wavelengths. The color bar has units of $\mathrm{m} / \mathrm{s}$

\subsection{The ATHENA-OAWL Mission}

Building on the years of system development and system studies, Ball together with the University of Maryland, worked mission formulation and engineering designs for the ATHENA-OAWL mission concept, proposed to NASA's Earth Venture Instrument - 2 (EVI-2) Announcement of Opportunity in 2013.

ATHENA-OAWL is a focused science mission to measure tropospheric winds along two lines of sight, and to provide concurrent information about tropospheric aerosols. The ATHENA-OAWL Science Objectives are to:

1. Create a breakthrough in prediction and understanding of low and mid-latitude weather and climate via improved sampling of wind profiles.

2. Improve our understanding of the effects of the interaction between aerosol radiative forcing and atmospheric dynamics on the genesis and lifecycle of tropical cyclones via collocated aerosol and wind measurements in the tropic.

3. Improve our understanding of the impact of long-range dust transport and aerosol transport on global energy and water cycles, air quality, and climate via collocated aerosol and wind measurements

Based on the ISS orbit (Figure 2), ATHENAOAWL would provide quasi-uniform sampling over $>80 \%$ of the Earth's surface, focusing on the low and middle latitudes where scientific demand for additional wind profiles is strongest. Based on conservative aerosol loading profiles, measurements are expected throughout the troposphere and lower stratosphere, with sufficient returns to provide space-based line of sight wind speed estimates with $<3 \mathrm{~m} / \mathrm{s}$ precision. This precision and coverage meets WMO requirements for Numerical Weather Prediction (NWP) and climate reanalysis applications.

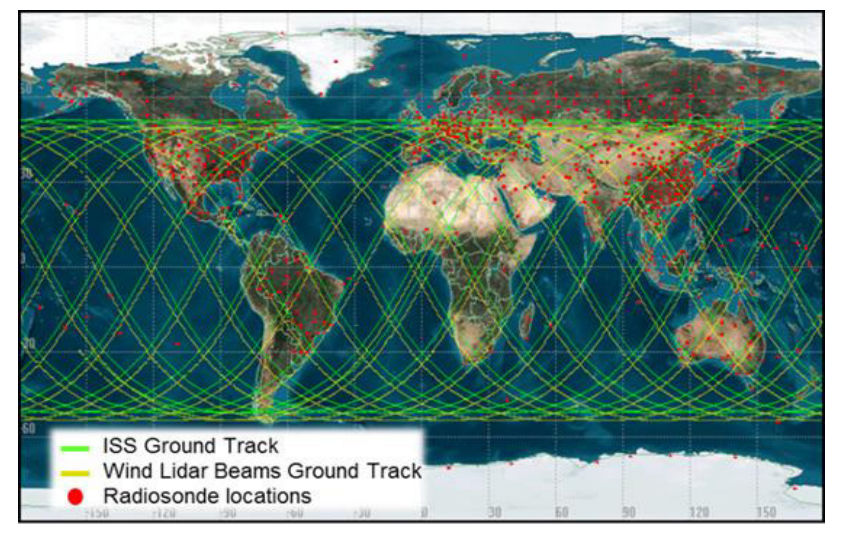

Figure 2. Current radiosonde launch locations (2 launches per day, at locations of red dots) compared to 24 hours of ISS orbit track.

By having two fixed, azimuthally-orthogonal looks at 40 degrees off nadir, the system can provide information about both components of the horizontal wind, reducing uncertainty in 
assimilation to NWP. Because the EVI mission opportunities are cost constrained, the team took a design to cost approach: build the system with the most science impact possible within the $\$ 94 \mathrm{M}$ cost cap (including $25 \%$ reserves). This mission approach is made possible in part by using the International Space Station (ISS), its Mission Operations, and the TDRSS downlink. ATHENA-OAWL can be a medium risk mission within the cost cap by leveraging heritage designs from CALIPSO and other space-based instruments built at Ball. The mission lifetime is 1 year (with a two year goal) and follows NASA Class C (Medium to Low Complexity) requirements.

\subsection{Technological Readiness}

The two critical subsystems for ATHENA-OAWL that require advanced development and spacequalification are the OA interferometer, and the narrowband $532 \mathrm{~nm}$ laser.

Interferometer Receiver: Prior to the proposal submission, Ball developed designs for an athermal version of the OAWL interferometer, building on heritage athermal optical designs that have already flown in space to ensure that orbital variations in temperature would not affect the interferometer alignment.

Laser: Fibertek Inc. has developed a "High Energy UV Demonstrator (HEUVD)" laser under a NASA SBIR Phase 3, and is now in final assembly. Prior to installation of the final tripling stage (used to generate the $355 \mathrm{~nm}$ UV light) this system first generates $532 \mathrm{~nm}$ light, allowing the system to be used for $532 \mathrm{~nm}$ testing and spacequalification. The output at $532 \mathrm{~nm}$ is $170 \mathrm{~mJ}$ at $150 \mathrm{~Hz}$ pulse repetition frequency for $26 \mathrm{~W}$ average optical power (compared to CALIPSO's 4.4 W split between 532/1064 $\mathrm{nm}$ ). These energy levels were chosen such that, when combined with ATHENA-OAWL's beam divergence, the system achieves the same eye-safety levels at the Earth surface from ISS as achieved for CALIPSO from its higher orbit.

The laser's opto-mechanical design is traceable to CALIPSO (and to the recent ICESat II build). The contamination control and de-rating approach were developed and validated on CALIPSO which has completed over 8 Billion pulses and 9 years of continuous operation.

\section{RESULTS AND ONGOING EFFORT}

The ATHENA-OAWL mission was not selected for the 2013 EVI-2 award. Two awards were made: Global Ecosystem Dynamics Investigation GEDI (Univ. of Maryland), and the Ecosystem Spaceborne Thermal Radiometer Experiment on Space Station ECOSTRESS (JPL). ATHENAOAWL was rated Category 3, however, and subsequently selected for risk reduction, Venture Technology development funding to advance its Technological Readiness Level (TRL). Under the current development effort, ATHENA-OAWL Venture-Tech (AOVT), Ball, in coordination with scientists at the University of Colorado, will demonstrate and provide validation for a twolook, $532 \mathrm{~nm}$ wavelength "Green-OAWL" (GrOAWL) airborne system. Under AOVT, Ball will upgrade existing OAWL instrument to match the ATHENA-OAWL two-look, two laser configuration and then perform new aircraft testing on the NASA WB-57 high altitude aircraft.

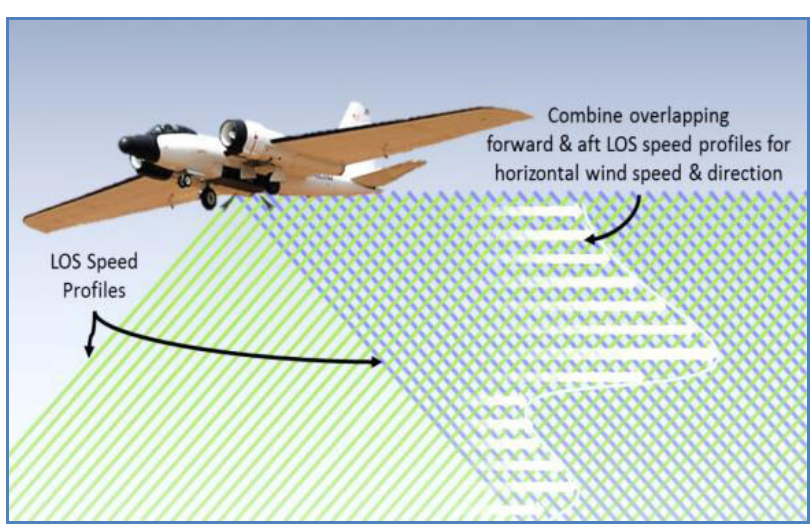

Figure 3. Example of the "GrOAWL" two-look aircraft flight configurations for measuring winds at two azimuthally separated angles. Forward beams are shown in green and aft facing beams are shown in blue. Due to the system geometry, beams have constant overlap enabling variable processing.

The system is currently in the design/build phase in preparation for demonstration flights scheduled for spring 2016. Balloon-sonde and drop sonde comparisons are planned for wind validation for different atmospheric conditions. Validation efforts will also incorporate comparisons with AERONET for aerosol calibration and radiometric performance validation.

The AOVT effort will also include spacequalification testing of key new technologies including $532 \mathrm{~nm}$ testing of the aforementioned 
HEUVD laser at Fibertek and thermal/vacuum testing of the new athermal interferometer, to advance their TRLs.

In addition to the AOVT effort, the "HSRL for Aerosols Winds and Clouds" (HAWC-OAWL) IIP, is currently funded by NASA ESTO to develop a dual-wavelength high spectral resolution lidar + winds version of OAWL for atmospheric transport and "Chemical Weather" studies. The dual-wavelength demonstrations and validation effort will take place after the GrOAWL test flights.

\section{CONCLUSIONS}

In an effort to address the need for the missing wind profile information over the tropics and midlatitudes, the ATHENA-OAWL Mission was proposed to the Earth Venture Instrument 2013 call. While the mission was not awarded, Venture Technology funding is enabling the build of the ATHENA-OAWL airborne GrOAWL system to prove out the two-look OAWL approach for a future space-based Doppler lidar technology demonstration. NASA WB-57 flight tests of the system with a focus on wind and performance validation, will take place in spring 2016.

\section{ACKNOWLEDGEMENT}

The authors wish to acknowledge the support of NASA's Earth Science Technology Office Instrument Incubator and Advanced Component Technology programs in making the OAWL demonstrations possible.

\section{REFERENCES}

[1] Huffaker, R.M. editor, "Feasibility Study of Satellite-Borne Lidar Global Wind Monitoring System," NOAA Technical memorandum ERL WPL-37, September 1978,

[2] Baker, W.E., R. Atlas, C. Cardinali, A. Clement, G. D. Emmitt, B. M. Gentry, R. M. Hardesty, E. Källén, M. J. Kavaya, R. Langland, Z. Ma, M. Masutani, W. McCarty, R. B. Pierce, Z. $\mathrm{Pu}, \mathrm{L}$. P. Riishojgaard, J. Ryan, S. Tucker, M. Weissmann, and J. G. Yoe, 2014: Lidar-Measured Wind Profiles: The Missing Link in the Global Observing System. Bull. Amer. Meteor. Soc., 95, 543-564.

[3] Atlas, R., R. N. Hoffman, Z. Ma, G. D. Emmitt, S. A. Wood, Jr., S. Greco, S. Tucker, L.
Bucci, B. Annane, M. Hardesty, and S. Murillo, 2015a: Observing system simulation experiments (OSSEs) to evaluate the potential impact of an optical autocovariance wind lidar (OAWL) on numerical weather prediction. $J$. Atmos. Oceanic Technol., submitted.

[4] Reitebuch, O., 2012a: Wind lidar for atmospheric research. In Schumann U. (Ed.): Atmospheric Physics - Background, Methods, Trends. Springer Series on Research Topics in Aerospace. ISBN 978- 916 3-642-30182-7, 487507

[5] Reitebuch, O., 2012b: The space-borne wind lidar mission ADM-Aeolus. In Schumann U. (Ed.): 918 Atmospheric Physics - Background, Methods, Trends. Springer Series on Research Topics in Aerospace, 815-827

[6] Schwiesow and Mayor, 1995: Coherent Optical Signal Processing for a Doppler Lidar Using a Michelson Interferometer," Coherent Laser Radar Conference (Keystone, CO), OSA Technical Digest Series 19.

[7] Grund, C., M. Lieber, B. Pierce, M. Stephens, C. Weimer, 2008: Optical Autocovariance Wind Lidar (OAWL) for Efficient Space-Based DirectDetection High-Resolution Aerosol Backscatter Winds, $24^{\text {th }}$ International Laser Radar Conference, Boulder, CO., 2008.

[8] S. C. Tucker, C. Weimer, 2013: Comparing and contrasting the Optical Autocovariance Wind Lidar (OAWL) and coherent detection lidar, Coherent Laser Radar Conference, Barcelona, Spain 17 - 20 June, 2013.

[9] Liu, Z., and T. Kobayashi, 1996: Differential discrimination technique for incoherent Doppler lidar to measure atmospheric wind and backscatter ratio. Opt. Rev., 3, 47-52

[10] Bruneau, D., 2001: Mach-Zehnder interferometer as a spectral analyzer for molecular Doppler wind lidar. Appl. Opt., 40, 391-399

[11] Bruneau, D., and J. Pelon, 2003: Simultaneous measurements of particle backscattering and extinction coefficients and wind velocity by lidar with a Mach-Zehnder interferometer: Principle of operation and performance assessment. Appl. Opt., 42, 11011114 